\title{
Teaching Process of Organic Chemistry with the Aid of Scientific Research Achievements
}

\author{
- Synthesis of Tetraindeno-Fused Spirofluorene Derivatives
}

\author{
Debin Xia* \\ School of Chemistry and Chemical Engineering \\ Harbin Institute of Technology \\ Harbin, China \\ xia@hit.edu.cn
}

\author{
Yulin Yang \\ School of Chemistry and Chemical Engineering \\ Harbin Institute of Technology \\ Harbin, China \\ ylyang@hit.edu.cn
}

\begin{abstract}
Fundamental organic chemistry is a compulsory course for college students major in chemistry. It is very difficult for teachers attracting students' attention for long term study on the bas is of the current teaching system. We introduce scientific research achievements, frontier knowledges and hot research topics into this lesson. Students' study interests are inspired dramatically. In detail, four types of organic reactions, namely Suzuki-Miyaura reaction, hydrolysis reaction, Friedel-Crafts acylation, and Knoevenagel condensation are illustrated, including their reaction mechanisms and reaction conditions. Then the common purification approaches and characterization methods are also presented. Considering the significance of $\mathrm{H}$ NMR in scientific research, it is emphasized, which is also an important knowledge point in the text book. Synthesis, purification and characterization are three key elements of organic chemistry, which are systematically combined and used in the teaching process. We believe the way presented in the article can also favor other teachers achieving the positive teaching effects.
\end{abstract}

Keywords-organic chemistry; teaching method; scientific research achievements; college education

\section{INTRODUCTION}

Organic chemistry is one important course, while only presenting the knowledge from text book cannot attract students' attention. Thus, it is essential to combine other teaching methods favoring the organic teaching process.

Providing students with the chance to learn cutting-edge technology is one of the effective ways to comprehend knowledge points and increase their study interests. Undoubtedly, introducing the scientific research achievements into organic teaching process is bound to promote the teaching effect. [1-3] However, the common task for college teachers is how to extract the effective research results and combines them with knowledge points effectively. We have some experience to present our own scientific research results related to organic chemistry to students, achieving a positive teaching effect, which we summarized in this article. Noted that the text book we mentioned is fundamental organic chemistry, 3rd edition authored by Qiyi Xing, Weiwei Pei, Ruiqiu Xu and Jian Pei. (Higher Education press)

\section{ORGANIC SYNTHESIS}

Conjugated small molecules has a significant influence on the performance characteristics of electronic devices, e.g., organic light emitting diodes (OLEDs) [4-5], organic fieldeffect transistors (OFETs) [6-7] and photovoltaic cells (PVs) [89]. These functional materials are key components for such kind of high technology.

During the teaching process, it is better to let students know that organic reaction is a tool and using this tool can construct many kinds of novel functional molecules. An example of scientific work [10] is introduced at the same time, especially the synthesis route to target molecules, as shown in scheme 1. The functional molecule Spiro-8CN can be prepared in an overall yield of 54\% using four types of reactions, including Suzuki-Miyaura reaction, hydrolysis reaction, Friedel-Crafts acylation, and Knoevenagel condensation. It is noted that all the four reactions are the knowledge points mentioned in the text book. Consequently, the students have a sense of achievement while understanding the knowledge points with scientific work. Furthermore, column chromatography is

Scheme 1. Syntheses of Spiro-4O and Spiro-8CN.
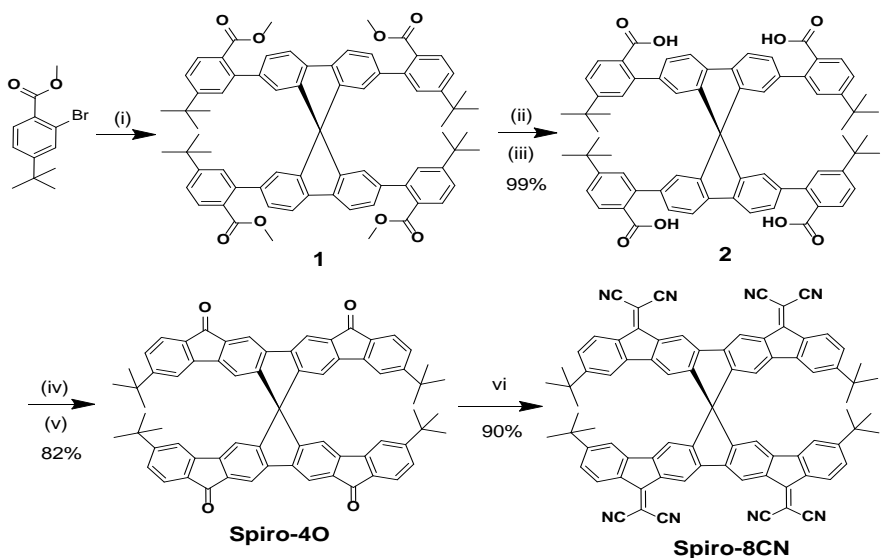

Reaction conditions: (i) 2,2',7,7'tetrakis(4,4,5,5-tetramethyl-1,3,2-dioxaborolan-2 yl)9,9'-spirobi[fluorene], $\mathrm{Pd}\left(\mathrm{PPh}_{3}\right)_{4}, \mathrm{~K}_{2} \mathrm{CO}_{3}$, toluene, $110^{\circ} \mathrm{C}$; (ii) $\mathrm{NaOH}$ ethanol reflux; (iii) $\mathrm{HCl}$; (iv) oxalyl chloride, $\mathrm{DMF}, \mathrm{CH}_{2} \mathrm{Cl}_{2}$, rt; (v) $\mathrm{AlCl}_{3}, \mathrm{CH}_{2} \mathrm{Cl}_{2}, 0^{\circ} \mathrm{C}$; (vi) $\mathrm{CH}_{2}(\mathrm{CN})_{2}, \mathrm{TiCl}_{4}$, Pyridine, $\mathrm{CH}_{2} \mathrm{Cl}_{2}$, rt. 
employed as an effective way to purify the target compounds, which expands students' knowledge regarding purifying process.

\section{A. Suzuki-Miyaura reaction}

The first knowledge point is Suzuki-Miyaura reaction, which is an organic reaction, classified as a coupling reaction, where the coupling partners are a boronic ester and an organohalide catalyzed by a palladium(0) complex. Akira Suzuki published this work in 1979 and won the 2010 Nobel Prize in Chemistry together with Richard F. Heck and Eiichi Negishi for their effort for developing palladium-catalyzed cross couplings in organic synthesis. Compound $\mathbf{1}$ was obtained in a yield of $74 \%$ via tetrafold Suzuki-Miyaura reaction between 2-bromo-4-(tert-butyl)benzoate and 2,2',7,7'tetrakis(4,4,5,5-tetramethyl-1,3,2-dioxaborolan-2-yl)-9,9'-

spirobi[fluorene] with the catalysis of tetrakis (triphenylphosphine)palladium( 0 ) and base of $2 \mathrm{M}$ aqueous potassium carbonate solution. Consequently, from the reaction mechanism to the background knowledge, we have a comprehensive understanding of this knowledge point.

\section{B. Hydrolysis of esters}

The second knowledge point is hydrolysis; one example is the hydrolysis of esters, which can be divided into basecatalyzed or acid-catalyzed hydrolysis. Regarding the former reaction mechanism, it is as follows: (1) the nucleophile (a nucleus-seeking agent, e.g., water or hydroxyl ion) attacks the carbon of the carbonyl group of the ester; (2) the intermediate collapses, reforming the carbonyl group results in the loss of the leaving group; (3) the formation of the carboxylic acid ends the hydrolysis. During this teaching process, we can also introduce the oldest commercially practiced example of producing soap. It is the hydrolysis of a fat with an aqueous base such as sodium hydroxide. During the process, glycerol is formed, and the fatty acids react with the base, converting them to salts. These salts are called soaps, commonly used in households. Going back to the research work, we employed a solution of sodium hydroxide as base, which was dropwised into a suspension of tetraesters $\mathbf{1}$. The obtaining residue was acidified with concentrated hydrochloric acid, obtaining white solid product with the yield of $99 \%$ after filtration.

\section{Friedel-Crafts acylation}

Friedel-Crafts acylation involves the acylation of aromatic rings, like thiophene, benzene etc. Typical acylating agents are acyl chlorides. Typical Lewis acid catalysts are acids and aluminium trichloride. Noted that these kinds of reactions normally occur in dry atmosphere. According to this reaction condition from the text book, tetrafold intramolecular FriedelCrafts acylation was achieved by treatment of compound 2 with oxalyl chloride and DMF. Even though the solubility of tetraketone Spiro-4O is not good, it can be purified by column chromatography with the isolated yield of $82 \%$ as an orange solid. The eluent of dichloromethane and hexane were employed.

\section{Knoevenagel condensation}

A Knoevenagel condensation is a nucleophilic addition of an active hydrogen compound to a carbonyl group, leading a dehydration reaction in which a molecule of water is eliminated. This type reaction is an important knowledge point in the chapter 15 (Carbon Anion and Condensation Reaction). Employing this reaction condition, malononitrile served as active hydrogen compound, the target product Spiro-8CN could be obtained under argon atmosphere in $90 \%$ yield with the assistance of Lehnert reagent.

\section{CHARACTERIZATION METHODS}

The characterization methods for organic compounds are an essential chapter for fundamental organic chemistry. Among them, H-NMR is the very common method, which needs students to study. In combine with our published scientific work, structures of Spiro-4O and Spiro-8CN are unambiguously confirmed by NMR spectroscopy. As shown in Fig. 1, the assignment of all aromatic protons is obtained by a combined analysis of ${ }^{1} \mathrm{H}-\mathrm{NMR}$, two-dimensional NMR. The ${ }^{1} \mathrm{H}$-NMR spectra reveal the symmetry of both molecules and the relative strength of electron-withdrawing moieties. Due to the stronger deshielding effect, protons $\mathrm{H}_{\mathrm{A}}$ and $\mathrm{H}_{\mathrm{B}}$ of Spiro-4O and Spiro-8CN resonate at lower magnetic field than $\mathrm{H}_{C}, \mathrm{H}_{\mathrm{D}}$ and $\mathrm{H}_{\mathrm{E}}$. Switching from carbonyl to dicyanovinylene units leads to an impressive deshielding effect for $\mathrm{H}_{\mathrm{A}}$ and $\mathrm{H}_{\mathrm{B}}$ of Spiro-8CN with chemical shifts of $8.98 \mathrm{ppm}$ and $8.22 \mathrm{ppm}$,

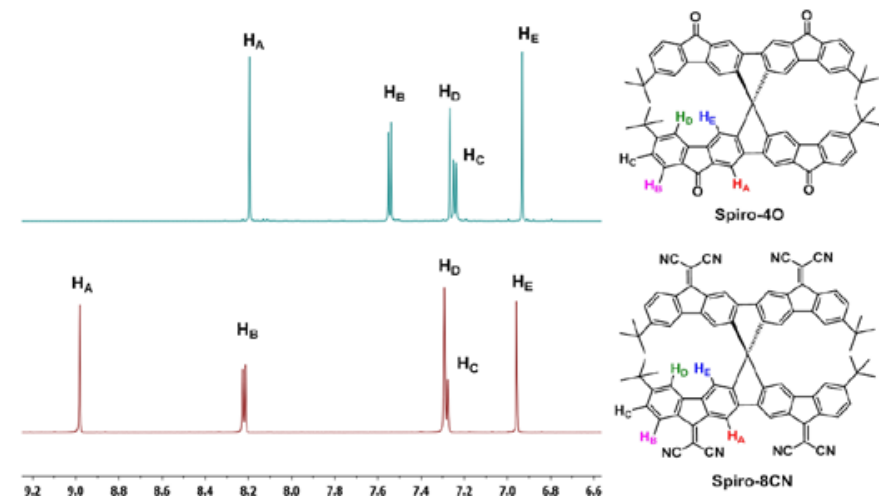

Fig. 1. Aromatic region of the ${ }^{1} \mathrm{H}$ NMR spectra of S piro-4O (top) and Spiro8CN (bottom).



Fig. 2. X-ray diffraction structure of Spiro-40. Left: molecular stacking pattern with a $\pi-\pi$ stacking distance of $3.35 \AA$. Right: intermolecular distances a: $3.32 \AA(\mathrm{C} \cdots \mathrm{C})$; b: $3.32 \AA(\mathrm{C} \cdots \mathrm{C})$; c: $3.16 \AA(\mathrm{C} \cdots \mathrm{O})$. Hydrogen atoms are omitted for clarity. 
respectively. On the basis of above mentioned discuss, it is much easier for students to apply the H-NMR knowledge they learned to scientific research results, which favors they have the sense of achievements.

Apart from that, single crystal analysis is another effective way to characterize molecules. Even though this technique is not included in the text book, while it is significant in the scientific research. We tried to present this way to students, arousing their study interest. As shown in Fig. 2, they not only present the molecular configuration but also the intermolecular interaction mold. This is the easy way for students to better understand molecular structure and how they interact with each other in the solid state.

\section{ULTRAVIOLET SPECTRUM}

Ultraviolet spectrum is one of the knowledge points in the chapter V. In this part, students need to understand the mechanism and the functionality of ultraviolet spectrum. The factors affect absorption of ultraviolet spectrum are also needs to be considered.

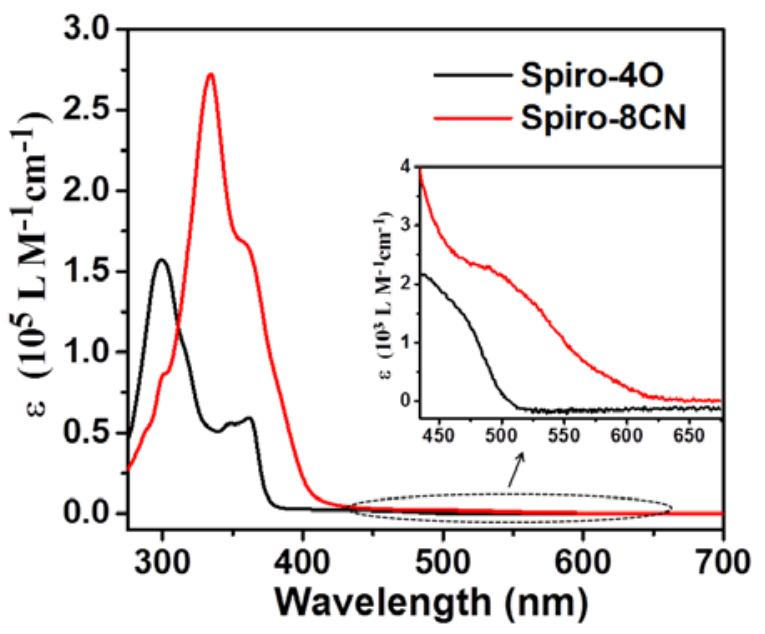

Fig. 3. UV-Vis absorption spectra of S piro-40 (black line) and S piro-8CN (red line) in $\mathrm{CH}_{2} \mathrm{Cl}_{2}$. The inset shows the UV-Vis spectra in the region 440$680 \mathrm{~nm}\left(\mathrm{n}-\pi^{*}\right.$ transitions $)$

In combine with the scientific work, as shown in Fig. 3, the UV-Vis absorption spectra recorded in dichloromethane. These two molecules provide similar absorption bands. From this part, we introduce to students that the optical properties of these two molecules are decided by their structures. To further explain the formation of the bands to students, we combined with the molecular orbital theory. The $\pi-\pi^{*}$ transition of the bisindenofluorene units cause the absorption bands in the range of $280-400 \mathrm{~nm}$, which is a significant bathochromic shift compared to that of fluorenone. The reason can be elucidated by the enhanced $\pi$-conjugation of the molecular backbone and the introduction of the electron-withdrawing groups. From Spiro-4O to Spiro-8CN, the absorption band redshifts significantly. This is mainly attributed to the decrease of the lowest unoccupied molecular orbital levels, ascribing to the stronger electron-withdrawing nature of dicyanovinylene versus carbonyl moieties. In the end, optical gaps estimated from the absorption edge of the solution spectra are $2.46 \mathrm{eV}$ (Spiro-4O) and $2.02 \mathrm{eV}$ (Spiro-8CN).

On the basis of above mentioned explanation, students can effectively grasp the related knowledge of ultraviolet spectrum, for instance, the formation reason of the absorption bands, the concept of extinction, $\pi-\pi^{*}$ or $n-\pi^{*}$ transitions, blueshift and redshift.

\section{ConClusion}

In conclusion, four types of reactions, namely SuzukiMiyaura reaction, hydrolysis reaction, Friedel-Crafts acylation, and Knoevenagel condensation were introduced. The knowledge points are well combined with the scientific work. Moreover, the characterization methods, especially the ${ }^{1} \mathrm{H}$ NMR is well illustrated, which enables students better grasp this method and increases their confidence to apply this tool. Lastly, ultraviolet spectrum is employed to characterize the optical properties of target compounds. The combination of scientific research achievements with teaching process is not only better for students to grasp their corresponding knowledge points also expand their vision. At the same time, this is also an effective way to boost student study and research interests. Finally, we believe the closed combination between the frontier scientific works and teaching process of fundamental organic chemistry is favoring students' learning effect.

\section{REFERENCES}

[1] X. Shi, "Penetration of scientific research achievements in the teaching process of organic chemistry," Un iversity Chemistry, China, vol. 30, pp. 26-31, October 2015 (In Chinese).

[2] H. Wu, J. Liu, S. Yang, D. Huang, S. Fan, "Practice of "Research" permeates in organic experimental teaching-a case of preparation for dye-sensitized solar cells,” Guangdong Chemical Industry, China, vol. 45, pp. 265-266, 2018 (In Chinese).

[3] M. Zhang, Q. An, D. Chen, L. Chen, "The integration of frontier scientific research achievements in organic chemistry teaching," Yunnan Chemical Technology, China, vol. 45, pp. 253-254, 2018 (In Chinese).

[4] C. Fan, C. Duan, C. Han, B. Han, H. Xu, "Dibenzothiophene sulfonebased phosphine oxide electron transporters with unique asymmetry for high-efficiency blue thermally activated delayed fluorescence diodes," ACS Applied Materials \& Interfaces, vol. 8, pp. 27383-27393, October 2016.

[5] D. Xia, B. Wang, B. Chen, S. Wang, B. Zhang, J. Ding, L. Wang, X. Jing, F. Wang, "Self-host blue-emitting iridium dendrimer with carbazole dendrons: nondoped phosphorescent organic light-emitting diodes," Angewandte Chemie International Edition, vol. 53, pp. 10481052, December 2013.

[6] D. Xia, T. Marszalek, M. Li, X. Guo, M. Baumgarten, W. Pisula, K. Muellen, "Solution-processible n-type organic semiconductors based on angular-shaped 2-(12H-dibenzofluoren-12-ylidene) malononitrile diimide,” Organic Letters, vol. 17, pp. 3074-3077, June 2015.

[7] S. Wang, M. Wang, X. Zhang, X. Yang, Q. Huang, X. Qiao, H. Zhang, Q. Wu, Y. Xiong, J. Gao, H. Li, “Donor-acceptor-donor type organic semiconductor containing quinoidal benzo[1,2-b:4,5-b']dithiophene for high performance N-channel field-effect transistors," Chemical Communications, vol. 50, pp. 985-987, October 2013.

[8] C. Wu, Y. Liu, C. Duan, Q. Pan, J. Zhu, F. Hu, X. Ma, T. Jiu, Z. Li, Y. Zhao, "Highly conjugated three-dimensional covalent organic frameworks based on spirobifluorene for perovskite solar cell 
enhancement,” Journal of the American Chemical Society, vol. 40, pp. 10016-10024, August 2018.

[9] D. Xia, D. Gehrig, X. Guo, M. Baumgarten, F. Laquai, K. Muellen, “A spiro-bifluorene based 3D electron acceptor with dicyanovinylene substitution for solution processed non-fullerene organic solar cells," Journal of Materials Chemistry A, vol. 3, pp. 11086-11092, April 2015.
[10] D. Xia, X. Guo, M. Wagner, M. Baumgarten, D. Schollmeyer, K. Muellen, "Cruciform electron acceptors based on tetraindeno-fused spirofluorene,” Crystal Growth \& Design, vol. 17, pp. 2816-2821, May 2017 